\title{
Meta
}

Journal des traducteurs

Translators' Journal

\section{BOULANGER, Jean-Claude (dir.) (1992) : Dictionnaire québécois d'aujourd'hui, Montréal, Dicorobert Inc., 1269 p. et 343 p.}

\section{Marthe Faribault}

Volume 38, numéro 3, septembre 1993

URI : https://id.erudit.org/iderudit/002723ar

DOI : https://doi.org/10.7202/002723ar

Aller au sommaire du numéro

Éditeur(s)

Les Presses de l'Université de Montréal

ISSN

0026-0452 (imprimé)

1492-1421 (numérique)

Découvrir la revue

Citer ce compte rendu

Faribault, M. (1993). Compte rendu de [BOULANGER, Jean-Claude (dir.) (1992) :

Dictionnaire québécois d'aujourd'hui, Montréal, Dicorobert Inc., 1269 p. et 343

p.] Meta, 38(3), 547-553. https://doi.org/10.7202/002723ar

Ce document est protégé par la loi sur le droit d'auteur. L'utilisation des services d'Érudit (y compris la reproduction) est assujettie à sa politique d'utilisation que vous pouvez consulter en ligne.

https://apropos.erudit.org/fr/usagers/politique-dutilisation/
Cet article est diffusé et préservé par Érudit.

Érudit est un consortium interuniversitaire sans but lucratif composé de l’Université de Montréal, l’Université Laval et l’Université du Québec à Montréal. Il a pour mission la promotion et la valorisation de la recherche. https://www.erudit.org/fr/ 
BOULANGER, Jean-Claude (dir.) (1992): Dictionnaire québécois d'aujourd'hui, Montréal, Dicorobert Inc., 1269 p. et 343 p.

Le Dictionnaire québécois d'aujourd' hui (dorénavant DQA) est une adaptation en profondeur - je reviendrai plus loin sur cette question - du Dictionnaire d'aujourd" hui, publié par la maison Robert en 1991. L'ouvrage se présente en deux parties principales et deux parties secondaires. La première partie, de 1269 pages, porte sur la langue générale et comprend environ 40000 entrées; à titre de comparaison, le Petit Robert a plus de 2000 pages et contient environ 60000 entrées. La seconde partie, de 343 pages, présente 12000 noms propres relevant de l'histoire, de la géographie et de la culture générale. En 
complément, on trouve une partie centrale, hors pagination, contenant un mini-atlas et des tableaux chronologiques, et, en fin de volume, des annexes grammaticales, de 52 pages, intitulées: Tableau des nombres, Petit dictionnaire des suffixes français, Tableau des conjugaisons, Dérivés des noms de personnes (réelles, mythologiques, imaginaires) et Noms et adjectifs (gentilés) tirés des noms propres de lieux (toponymes).

La facture de l'ensemble pourrait rappeler le Petit Larousse, avec ses deux parties, langue et noms propres, et ses célèbres pages centrales roses. Mais la comparaison s'arrête là, tant pour la forme que pour le contenu.

Dans le détail, la seconde partie du dictionnaire présente: 1) des noms de lieux, dont toutes les agglomérations québécoises de plus de 5000 habitants; 2) des noms de personnes ayant œuvré dans le monde de la littérature (Michel Tremblay), des arts visuels (Jean-Paul Lemieux), de la musique (Gilles Vigneault), des sciences et des techniques (le Frère Marie-Victorin, Joseph-Armand Bombardier), du journalisme (André Laurendeau), de la politique (Jean Lesage), des sports (Maurice Richard), etc.; 3) des «mots-noms», comme Humanistes, Lumières, Révolution tranquille, à côté de Révolution française. Cette seconde partie encyclopédique est complétée par une série de documents présentés dans la partie centrale non paginée du dictionnaire. On y trouve: 1) un mini-atlas de 65 cartes, dont 19 concernent uniquement la géographie du Canada et du Québec;2) une chronologie allant de la Préhistoire à nos jours, dans laquelle l'information est répartie en cinq colonnes : religion et philosophie, histoire politique, sciences et techniques, littératures, arts et musique; 3 ) des listes se rapportant plus particulièrement à l'histoire canadienne et québécoise: Gouverneurs, intendants, évêques de la Nouvelle France; Gouverneurs de Québec avant la Confédération; Gouverneurs généraux, premiers ministres du Canada; Lieutenants-gouverneurs, premiers ministres du Québec; Canonisés et béatifiés canadiens.

On peut se féliciter de disposer dorénavant d'un répertoire facilement accessible pour les noms propres relatifs à la culture française de l'Amérique du Nord. On peut cependant reprocher à ce répertoire de faire encore trop de place aux noms propres relatifs à la culture mondiale en général et française en particulier. Il aurait peut-être été préférable de donner un répertoire entièrement français nord-américain, en considérant qu'un tel répertoire existe déjà sous forme d'une publication autonome (Veyron, Michel, Dictionnaire canadien des noms propres, Montréal, Larousse Canada, 1989) et que l'équivalent mondial et français est déjà largement répandu (Petit Robert 2, Dictionnaire universel des noms propres).

Bien entendu, c'est la première partie portant sur la langue française qui constitue le cœur de ce dictionnaire, et le cœur de la polémique que sa publication a déclenchée. Je soulignais au début qu'il s'agissait d'une adaptation en profondeur du Dictionnaire d'aujourd' hui. Pour être plus exact, il faudrait dire qu'il s'agit d'un travail original, qui s'appuie sur le même fonds documentaire que le Dictionnaire d'aujourd' hui. Autrement dit, ce n'est pas un dictionnaire français «maquillé de québécois», une sorte de Petit Robert «québécisé», mais bien un dictionnaire repensé en profondeur pour rendre compte de l'usage français nord-américain, principalement québécois. Il suffit d'ailleurs d'en feuilleter quelques pages, au hasard, pour constater qu'il ne ressemble à aucun de ses prédécesseurs. En effet: 1) le Dictionnaire général de la langue française au Canada de Louis-Alexandre Bélisle, publié à Montréal chez Beauchemin pour la première fois en 1957 et régulièrement réédité par la suite, se contentait de plaquer quelques «canadianismes»au dictionnaire de Littré-Beaujean ; 2) le Dictionnaire du français Plus, publié à Montréal au C.E.C. en 1988, poussait l'adaptation du Dictionnaire Hachette de la langue française jusqu'à québéciser les exemples, à inclure des citations d'auteurs québécois, à ajouter des renseignements de nature étymologique et encyclopédique; mais il n'allait pas jusqu'à québéciser la transcription phonétique des mots du français général, ni à marquer ou à 
éliminer les francismes (baba-cool), ni à inclure des mots un tant soit peu familiers du québécois (broue), pas plus que les anglicismes les plus courants (fun, gang, chum), ni à établir un lien entre usage québécois et usage français (broue en québécois correspond à mousse ou écume, suivant le cas); 3) le Dictionnaire des canadianismes de Gaston Dulong, publié à Québec et à Paris par Septentrion et Larousse en 1989, confectionné suivant une règle purement différentielle, se limitait à décrire la portion du lexique québécois qui n'appartient pas au français commun. Par rapport à ses trois prédécesseurs, le DQA pousse la québécisation très loin. La nomenclature a été enrichie d'environ 4000 mots d'entrée typiques du français nord-américain, principalement québécois, soit autant que dans le Dictionnaire du français Plus, mais en laissant une plus large part aux mots de niveau familier et aux anglicismes. La transcription phonétique est donnée suivant le système phonologique du québécois tant pour les entrées spécifiquement québécoises que pour les mots du français commun. Les exemples ont été adaptés au contexte nordaméricain. La structure même des articles du français fondamental a parfois été revue en fonction de l'usage linguistique nord-américain. La partie analogique du dictionnaire permet de passer d'un usage québécois à son ou ses équivalents en français commun et en franco-français, et vice-versa. Les formes féminines des titres et fonctions ont été systématiquement données, ce qui correspond à la tendance québécoise, à l'opposé de l'usage français beaucoup plus conservateur; ainsi, on trouvera à l'entrée «professeur» la remarque suivante: «L'O.L.F. propose professeure au féminin.»; et à l'entrée «plombier»: «L'O.L.F. propose plombière au féminin.»

On peut bien sûr ne pas être d'accord avec le choix de certaines entrées (twit) ou avec le fait de les présenter sans aucune marque d'usage globale (pogner), et se mettre à pousser les hauts cris, comme l'ont fait la plupart des détracteurs du DQA, tant dans la presse écrite que dans la presse électronique. Mais on se fera une idée plus juste de l'ensemble si on prend la peine d'examiner dans le détail une section du dictionnaire, audelà des seuls mots d'entrée et en tentant de faire abstraction de tout préjugé. Ainsi, parmi les québécismes de la lettre «L» (34 pages), on relèvera: une soixantaine de gentilés (labradorien, lachenois, lachinois, etc.), des formes marquées comme familières (lambin et lambiner, lastique, licher, lisable présenté sous lisible, etc.), des anglicismes marqués «Anglicisme» (lady, leader, leasing, loafer n.m., lobby, look), «Anglicisme familier» (lift, lousse) ou non marqués (lock-out, lunch), et enfin des technicismes (labrador, laveauto, lobo, logotype, L.S.D., ltée). En sous-entrée, on trouvera des dérivations de sens, dont certaines sont marquées comme familières (lanceur, terme de baseball, sous lancer; lard; laveuse, sous laver; libéral ; licence Fam.; ligne Fam., terme relatif à la cocaïne; liqueur Fam.), des exemples adaptés au contexte québécois (lac Mistassini, lac SaintJean, Grands Lacs, sous lac; sous lieu: «haut lieu: ...Le balcon de l'hôtel de ville de Montréal fut un des hauts lieux de la visite du général de Gaulle au Québec en 1967»), des expressions et locutions particulières au français nord-américain (tomber dans le lac, au sens d'échouer; laitue iceberg; gros lard, tête de lard, etc.). Dans la partie analogique, on trouvera de nombreux renvois vers les mots et expressions du français québécois, parfois marqués comme familier (barachois, sous lagune; taponneur, fam. têteur, sous lambin; niaiser, taponner, fam. gosser, têter, sous lambiner; fam. garrocher, sous lancer; magasinage, sous lèche-vitrines; clenche, sous loquet; fam. coq-l'ail, sous loucheur; Etre connu comme Barrabas dans la passion pour Etre connu comme le loup blanc, sous loup). Parallèlement aux matériaux québécois, certains usages franco-français ont été marqués comme tels (landau; esquimau au sens de «glace»; lessive). Enfin certains articles du français commun ont été réécrits pour rendre compte de l'usage québécois ; ainsi, dans l'article lancer n.m., le deuxième sens «Épreuve d'athlétisme...» est suivi d'un troisième sens, qui tient compte des domaines particuliers du hockey et du baseball. 
En définitive, l'examen de la section «L» laisse apparaitre une grande richesse de matériaux. On ne peut cependant s'empêcher de noter quelques lacunes au passage (sous laine, on trouve l'unité laine des Pyrénées, mais pas laine d'habitant; sous lieuse, on trouve la définition: «Machine servant à lier les gerbes», mais pas le sens québécois de «Corde servant à lier les gerbes»). On note également des incohérences de traitement des dérivés suffixaux; en effet, ceux-ci doivent en principe être traités en sous-entrée de la base à partir de laquelle ils ont été dérivés; or, un certain nombre d'entre eux ont une entrée autonome, sans raison apparente (largable et largage, de larguer; libérable, de libérer; louable, de louer). Enfin, l'application automatique de la règle lexicographique a mené dans un cas à un traitement discutable; on relève en effet sous limier la remarque suivante: «Le féminin limière est virtuel.» En fait, la forme féminine n'est pas attestée dans la langue non pas par conservatisme, mais bien parce que limier ne correspond à aucun titre ni fonction; le terme n'apparaît que rarement en dehors de l'expression figée fin limier. À ce compte-là, aumônier a pour féminin aumônière, et cette forme féminine restera virtuelle tant et aussi longtemps que le clergé sera exclusivement masculin!

Outre les corrections de détail signalées à l'examen de la section «L», on peut suggérer à l'équipe de rédaction, lors de la révision de l'ouvrage, de porter une attention particulière aux cinq points suivants: la transcription phonétique, le traitement des gentilés, la correction typographique, le discours métalinguistique et les articles grammaticaux.

La transcription phonétique actuelle ne tient pas compte de l'opposition phonologique, très vivante en québécois, entre / $\varepsilon /$ et / $\varepsilon$ : /, comme dans / metr / et / me:tr / (mettre et maitre). Les auteurs prennent position sur cette question dans l'introduction: «...ce n'est pas à un dictionnaire d'apprentissage général que revient la tâche de s'attaquer à cette description.» L'argument n'est guère convaincant.

Le double traitement des gentilés, en nomenclature et en annexe, encombre inutilement le corps du dictionnaire, d'autant plus que chaque article redonne la règle générale d'orthographe suivant laquelle le gentilé avec majuscule sert à désigner les natifs ou les habitants de la ville (Montréalais), de la région (Montérégien) ou du pays (Canadien). L'annexe des gentilés, précédée d'une remarque portant sur l'usage orthographique, aurait suffi amplement.

Il est normal de trouver un nombre relativement élevé de coquilles dans la première édition d'un dictionnaire, car le texte lexicographique est un texte extrêmement complexe, donc difficile à réviser. Le DQA n'échappe pas à cette fatalité; cependant, ce n'est pas une raison suffisante pour rejeter l'ouvrage du revers de la main. On peut simplement suggérer à l'équipe de rédaction de faire effectuer la révision de cette première édition par des Québécois, ce qui permettrait de corriger des erreurs dénotant une méconnaissance totale du parler québécois (campivalleusien pour campivallensien, bagasse pour bagosse), confirmée dans certains cas jusque dans la transcription phonétique (kãpivalòsje). De plus, il faudra porter une attention particulière aux mots rares (ou québécois) comprenant des $n$ ou des $u$, des $g$ ou des $q$ et des $a$ ou des $o$; il semble en effet que l'écriture manuscrite du ou des rédacteurs ait présenté ce genre de confusions, d'où des coquilles comme givint (relevé dans la partie analogique de laine) ou qivint (relevé dans la partie analogique de ovibos) pour qiviut ; également carasse pour carosse (dans la partie analogique de landau).

Les définitions doivent être révisées ici et là, afin de les rendre conformes à la règle lexicographique; par exemple la définition d'alléghanien, à corriger par «Relatif aux Alléghanys,...» ou «Des Alléghanys,...». La partie analogique devrait également être systématiquement testée, afin d'éviter que des renvois ne mènent à aucune entrée; par exemple, le renvoi quinquet, sous lampe. La rédaction des remarques (REM.) doit également être revue; par exemple, sous ouananiche et sous ouaouaron, on relève: «Dans ce 
mot d'origine amérindienne, l'élision est rare», pour expliquer le fait qu'on dise «Le cri de l'ouaouaron» et «Pêcher l'ouananiche» ou «Le cri du ouaouaron» et «Pêcher la ouananiche»; il faudrait corriger par «Devant ce mot...». De même sous dépanneur, on lit: «REM. Le terme accommodation ne s'emploie pas au sens de «dépanneur»; il faut comprendre: «...ne devrait pas s'employer...». Enfin, l'orthographe d'accord devrait être revue dans les exemples, comme «Son collègue s'est fait lapidé....», à corriger en «lapider», dans l'article lapider.

$\mathrm{Si}$, à la seconde édition, on veut vraiment pousser la québécisation du dictionnaire jusqu'au bout, il faudrait donner, par exemple, les usages québécois des pronoms personnels (aux entrées il, elle, ils et elles en particulier) et les formes québécoises des interrogatifs qui, que, quoi, quand, comment, combien. Pour le moment, le seul article grammatical que j'aie pu repérer est celui du morphème de l'interrogatif - $t u$, caractéristique du québécois parlé. La porte est donc déjà ouverte à ce niveau de traitement.

Il reste maintenant à examiner les points névralgiques de cet ouvrage, soit le choix des mots d'entrée et le système des marques d'usage. Ce sont eux qui ont déclenché toutes les passions lors de la parution du dictionnaire.

On peut certes ne pas être d'accord avec l'inclusion d'une partie du lexique d'une langue dans un dictionnaire. C'était d'ailleurs la position des Académiciens lors de la parution du premier dictionnaire de l'Académie en 1694 face à une partie de l'usage familier et à tout le vocabulaire des sciences et des techniques, considéré comme appartenant au bas langage. À l'opposé, Antoine Furetière publiait en 1690 son Dictionnaire universel, contenant généralement tous les mots français tant vieux que modernes et les termes des sciences et des arts; sa position était celle d'un observateur de l'usage et non pas celle d'un censeur. Le résultat est que trois cents ans plus tard, le dictionnaire de Furetière est le meilleur instrument de travail lexicographique du XVII ${ }^{\mathrm{e}}$ siècle, tandis que le Dictionnaire de l'Académie de 1694 est relégué au rang des curiosités, monstrueuses dirions-nous. De la même façon, le Québec a connu, il y a quelques décennies, des versions du Petit Larousse expurgées de tous les termes relatifs à la sexualité; ces dictionnaires sont également relégués au rang de curiosités. Dans le cas qui nous occupe, entre le refus total de tout québécisme dans un dictionnaire général de la langue française et la québécisation poussée au maximum, toutes les positions sont possibles. Certains se sont maintenant habitués à la soixantaine de «canadianismes de bon aloi» introduits dans les Larousse et les Robert depuis les années 70 et voudraient qu'on en reste là. D'autres, plus ouverts, admettent en plus les termes désignant des réalités particulières au Québec ou à l'Amérique du Nord (assurance-maladie, A.A.). Par contre, la plupart rejettent l'immense majorité des anglicismes, identifiés depuis deux siècles comme le symbole même de la dégénérescence du français en Amérique du Nord. Enfin, presque tous tiquent devant les usages très familiers ou vulgaires (guidoune, plotte, crosser).

Mais, à partir du moment où l'on est d'accord avec le point de vue descriptif, et non prescriptif, et dans une perspective assez large pour permettre l'inclusion de portions du lexique sujettes à discussion, on concède au lexicographe deux choix : il peut donner tous les matériaux en vrac, tant ceux du français commun que ceux du franco-français ou du québécois, et laisser au bon jugement de l'utilisateur le choix des termes; il peut aussi marquer les usages. Les critiques du DQA laissent parfois entendre que les rédacteurs du dictionnaire ont opté pour la première solution. Or, rien n'est plus faux. En effet, les rédacteurs ont suivi la deuxième règle, comme ils l'expliquent dans l'introduction, et l'ont appliquée de façon cohérente dans le corps du dictionnaire. Ainsi, tous les termes, expressions et usages considérés comme n'appartenant pas au niveau correct, écrit ou oral, sont marqués familier, très familier ou vulgaire. Quant aux anglicismes, ils sont marqués anglicisme, lorsqu'ils sont sentis comme très usuels (hot chicken, smoked-meat), 
ou anglicisme familier, lorsqu'ils ne relèvent pas du niveau neutre ou courant (fun, slack); ils peuvent aussi faire l'objet d'une remarque, lorsque leur emploi est critiqué, ou encore ne pas être marqués du tout. Dans ce cas particulier des anglicismes, les défenseurs de la pureté de la langue française au Québec auraient voulu les voir tous critiqués, sans exception, et systématiquement marqués comme à proscrire, et non partiellement marqués comme familiers. C'est un point de vue hypercorrectif, qui n'est pas celui de ce dictionnaire. Pour l'utilisateur, il reste que tous les anglicismes sont marqués d'une façon ou d'une autre, sauf ceux qui sont intégrés au français commun (lock-out, lunch). Le seul traitement vraiment discutable à mon point de vue est celui des calques; ils n'ont pas été identifiés comme anglicismes, mais ont été néammoins marqués comme familiers; ils apparaissent donc dans le dictionnaire comme des dérivations de sens internes au français, comme par exemple balance, dans le sens de «ce qui reste...», et matériel, dans le sens de «tissu».

La catégorie de termes et d'expressions qui s'est attiré les critiques les plus véhémentes est constituée par les québécismes qui n'ont pas été marqués comme tels par les rédacteurs. Certains auraient voulu voir tous les québécismes, sans exception, marqués au moins comme familiers. Autrement dit, selon la logique de ce point de vue, un niveau de langue correct (et non soutenu) ne devrait laisser aucune place à tout élément n'appartenant pas au français commun. C'est un point de vue sur la norme qui a eu cours au Québec jusqu'au milieu des années 70; mais depuis les années 80, il est clair qu'une norme plus souple s'est implantée, distincte de la norme franco-française sans toutefois aller jusqu'au joual. Le problème ici revient encore à une question de degrés. Et, dans ce débat, on peut observer une stratification des réactions suivant l'âge; ainsi les 45 ans et plus sont plutôt tenants d'une norme strictement française; les 30-40 ans, qui ont vécu leur jeunesse dans les années 70 , à l'époque dite du «joual-fierté» et de l'affirmation de l'identité québécoise, sont plus facilement accueillants aux usages québécois; quant à ceux qui ont aujourd'hui 20 ans, ils se demandent qui croire: les plus vieux ou les moins vieux; ceux qui jappent le plus fort ou les plus amoureux de leur langue; les traducteurs, les linguistes ou les littéraires; leurs professeurs ou les journalistes?

En conclusion, cette question d'un dictionnaire québécois qui rende compte de l'usage réel de la langue française au Québec, tout en définissant une norme qui lui soit propre, fait et fera couler encore beaucoup d'encre et de salive. Quoi qu'il en soit, nous disposons dorénavant d'un outil de travail extrêmement précieux, parce qu'il est le seul à être véritablement descriptif de l'usage français nord-américain. Il se pose comme complément indispensable à ses prédécesseurs, qui demeurent utiles pour le point de vue historique sur le lexique québécois (Dictionnaire du français Plus) ou pour la portion du lexique québécois plus rare ou régional (Dictionnaire des canadianismes).

Les moins violents parmi les critiques de cet ouvrage ont dit qu'il s'agissait d'un instrument de travail pour spécialistes, qu'il fallait l'utiliser avec prudence, ne pas le mettre entre toutes les mains et, entre autres, pas entre celles des enfants qui ne sauront pas faire la part des choses. De laisser ainsi sous-entendre que les adultes non spécialistes n'auront pas le jugement nécessaire pour utiliser convenablement un tel ouvrage, c'est faire comme les politiciens canadiens qui, l'automne dernier, refusaient de diffuser le texte de l'accord de Charlottetown sous prétexte que le simple citoyen ne le comprendrait pas. On croit rêver quand on entend de tels propos dans un pays démocratique et hautement scolarisé! Quant aux mises en garde face au public scolaire, elles dénotent un manque de confiance profond dans les jeunes et une absence totale de conscience pédagogique, car à quoi sert l'école, si ce n'est à former le jugement des enfants et des adolescents?

Quel que soit le sort réservé à ce dernier-né des dictionnaires québécois, qu'il soit sanctionné par le ministère de l'Éducation du Québec ou non, que la meute des puristes 
réussisse à lui casser les reins ou non, il restera le seul instrument de travail qui permette aux étrangers de lire une bonne partie de la littérature québécoise. Il restera également le dictionnaire le plus complet et le plus utile pour toute personne qui apprend le français comme langue seconde au Canada; il sera un complément indispensable au Dictionnaire du français canadien de Sinclair Robinson et Donald Smith, publié à Toronto en 1990 (réédition du Manuel pratique du français canadien, publié en 1973), qui est en fait un lexique thématique trilingue «québécois» - «français commun» - «anglais». Il peut encore être utilisé comme instrument d'autocorrection par les locuteurs natifs du français québécois, jeunes et moins jeunes, car tous les termes de remplacement des québécismes en français commun sont donnés, soit par la définition elle-même, soit par le système des renvois. Autrement dit, bien plus qu'un danger pour les élèves, la publication de ce dictionnaire représente une occasion unique pour eux de réapprendre à utiliser intelligemment un dictionnaire et d'affiner leur performance linguistique en apprenant à maîtriser différents niveaux de langue : français commun, correct et familier, franco-français, québécois commun, correct et familier. Aux professeurs et aux parents de les aider à y parvenir.

Pour les traducteurs, l'utilité de ce dictionnaire est peut-être moins évidente. Les apprentis traducteurs pourront certes s'en servir comme instrument d'autocorrection, en parallèle avec les autres dictionnaires correctifs disponibles sur le marché. Cependant, ils devront se rappeler que ce dictionnaire ne leur donne pas de solution de correction pour tous les mots et usages que les rédacteurs n'ont pas marqués parce qu'ils les considèrent comme acceptables dans l'usage écrit correct du français québécois. Le problème réside dans le fait que, depuis plusieurs décennies, les traducteurs québécois et canadiens utilisent, enseignent, imposent et se voient imposer une norme très stricte du français écrit, d'où sont exclus systématiquement tous les anglicismes, tous les calques ou ce qu'on croit en être, tous les québécismes sauf les termes absolument nécessaires à la désignation des réalités nord-américaines, tous les emplois familiers ou populaires, sauf dans des cas bien particuliers. On peut alors comprendre que les traducteurs professionnels et les professeurs de traduction voient ce dictionnaire d'un assez mauvais oil, qu'ils aillent jusqu'à le rejeter. Mais, ce faisant, ils doivent se rappeler qu'ils jugent en tant que traducteurs soumis à une norme très stricte et non pas en tant qu'utilisateurs habiles et mesurés du français parlé et écrit au Québec. 